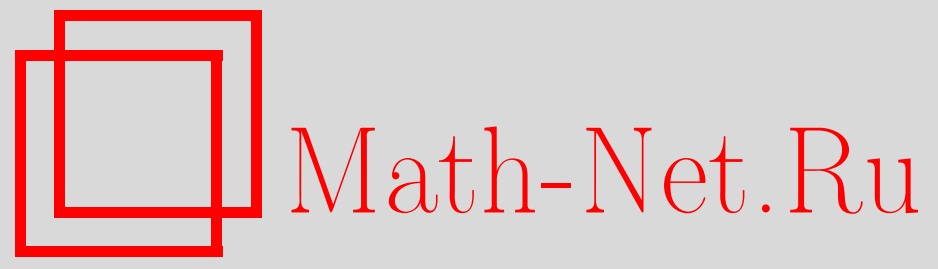

Д. А. Жуков, Об одном классе клеточных схем, Дискрет. матем., 2006, том 18, выпуск 4, 84-98

DOI: https://doi.org/10.4213/dm81

Использование Общероссийского математического портала Math-Net.Ru подразумевает, что вы прочитали и согласны с пользовательским соглашением http://www . mathnet.ru/rus/agreement

Параметры загрузки:

IP : 34.227 .88 .159

26 апреля 2023 г., 12:05:10

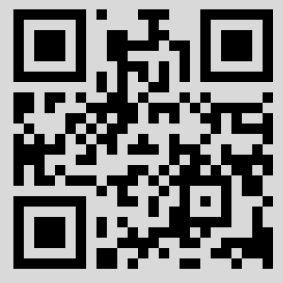


УдК 519.7

\title{
Об одном классе клеточных схем
}

\author{
๑) 2006 г. Д. А. Жуков
}

\begin{abstract}
В работе предложен класс клеточных схем, $T$-схем, для которого удалось связать нижнюю оценку площади с глубиной: чем меньше глубина схемы, тем больше должна быть ее площадь. Приведены примеры $T$-схем логарифмической глубины для задач вычисления $n$ префиксных сумм, суммы и разности двух $\boldsymbol{n}$-разрядных чисел. Показано, что площадь этих схем есть $O(n \log n)$ и оптимальна по порядку.
\end{abstract}

\section{1. Введение}

Клеточная схема $[4,11]$ имеет вид прямоугольника на плоскости, составленного из клеточных элементов. Будем называть схему правильной, если все ее входы и выходы расположены по краям. Предполагается, что все клеточные схемы в этой работе правильные. Под сложностью схемы понимается занимаемая ею площадь. Будем считать, что длина клеточной схемы измеряется по горизонтали, а ширина - по вертикали. Длину схемы $S$ обозначим $l(S)$, а ширину - $h(S)$. Тогда площадь $A(S)$ схемы $S$ равна $l(S) h(S)$.

Клеточный элемент имеет вид единичного квадрата. На его сторонах расположены входы и выходы, не более одного на каждой стороне. Клеточный элемент назовем функциональным, если он реализует нетождественную функцию, и коммутационным, если он реализует тождественные функции. Кроме функциональных и коммутационных элементов, имеется изолирующий клеточный элемент без входов и выходов. Предполагается, что клеточные элементы реализуют функции $k$-значной логики и каждая функция из $P_{k}$, существенно зависящая не более чем от двух переменных, реализуется некоторым функциональным элементом. Используемый далее базисный набор элементов изображен на рис. 1. В верхнем ряду приведены три функциональных элемента (реализующие константу $c$, одноместную функщию $g \in P_{k}$ и двуместную функцию $f \in P_{k}$ ) и изолирующий элемент. В нижнем ряду представлены коммутационные элементы. При построении схемы допускаются повороты клеточных элементов на углы, кратные $\pi / 2$.

Цепью в клеточной схеме назовем всякую последовательность клеточных элементов, в которой выход каждого элемента, кроме последнего, соединен с входом следующего. Длиной цепи будем называть число содержащихся в ней функциональных элементов. Цепь наибольшей длины среди цепей, соединяющих входы и выходы схемы, назовем максимальной. Глубиной клеточной схемы назовем длину ее максимальной цепи. Глубину схемы $S$ будем обозначать $d(S)$. С. С. Кравцов [4] показал, что для любой булевой функции $n$ аргументов можно построить реализующую ее клеточную схему площади $\lesssim(9 / 2) 2^{n}$, и что для почти всех функщй $n$ аргументов площадь их реализации клеточными схемами не менее $(1 / 4) 2^{n}$. Позднее А. Альбрехт [1] установил, что функция 


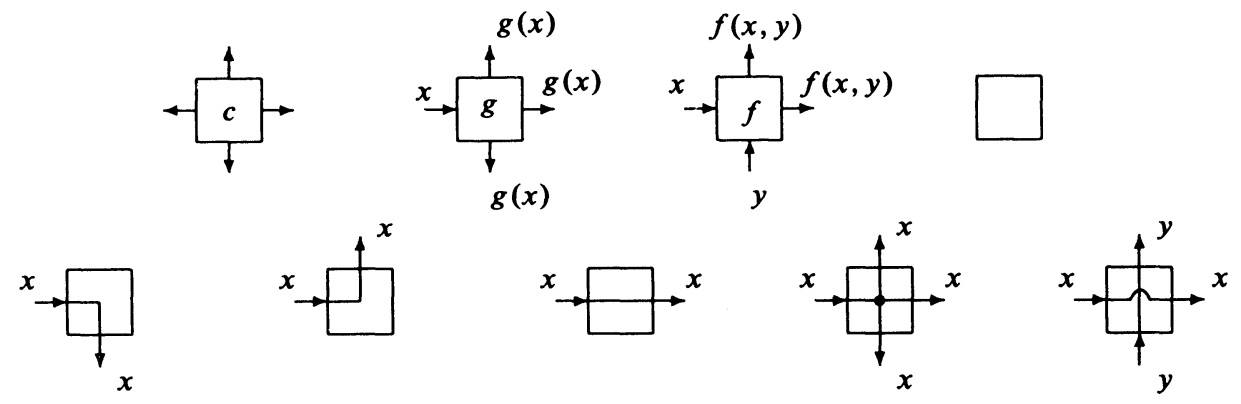

Рис. 1. Базисные клеточные элементы

Шеннона

$$
A(n)=\max _{f} \min _{S} A(S),
$$

где максимум берется по всем булевым функциям $f$ от $n$ переменных, а минимум - по всем вычисляющим $f$ клеточным схемам $S$, имеет асимптотику

$$
A(n) \sim \sigma 2^{n}
$$

где константа $\sigma$ удовлетворяет неравенству

$$
\frac{1}{4} \leqslant \sigma \leqslant \frac{9}{2} .
$$

Нижние оценки в этих неравенствах установлены мощностным методом [7]: число схем меньшей площади меньше числа всех булевых функций $n$ аргументов.

Всякую клеточную схему можно рассматривать как укладку некоторой схемы из функциональных элементов в прямоугольную решетку. Произвольный узел решетки может либо не принадлежать схеме, либо быть функциональным элементом, либо быть вершиной пути, соединяющего какой-то вход (схемы или ее функционального элемента) с какимто выходом (схемы или ее функционального элемента). Если базисный набор клеточных элементов содержит реализующий две тождественные функции коммутационный элемент (рис. 1, крайний справа в нижнем ряду), то через не являющийся функциональным элементом узел решетки допускается прохождение не более двух путей из укладки. Такая укладка в [5] названа 2-вложением. Если же все базисные коммутационные элементы реализуют не более одной тождественной функции, то через узел решетки может проходить не более одного пути (1-вложение [5]). Укладки схем везде ниже будут правильными 2-вложениями. Очевидно, что длина и ширина укладки равны длине и ширине схемы.

Укладкам графов в прямоугольные решетки посвящена обширная литература (см., например, обзор в монографии Дж. Ульмана [8]). Универсальные нижние оценки площади как правило используют обилие ребер графа схемы. Известны нетривиальные результаты и для разреженных графов, в частности, для деревьев.

Рассмотрим плоскую область $D$, у которой на границе $\partial D$ выбраны $n$ точек $x_{1}, \ldots x_{n}$ с попарным расстоянием, не меньшим единицы. В [13] доказано, что если $x_{1}, \ldots x_{n}-$ листья полного двоичного дерева $T$, лежащего на плоскости внутри $D$, а область $D$ выпуклая, то сумма длин ребер дерева $T$ не меньше чем $\Omega(n \log n)$. Заметим, что в статье используются следующие обозначения $O, \Omega$ и $\Theta: f(n)=O(g(n))$, если найдутся 
постоянная $c>0$ и число $n_{0}$ такие, что для всех $n \geqslant n_{0}$ справедливо неравенство $0 \leqslant f(n) \leqslant c g(n) ; f(n)=\Omega(g(n))$, если и только если $g(n)=O(f(n)) ; f(n)=\Theta(g(n))$, если и только если $f(n)=O(g(n))$ и $f(n)=\Omega(g(n))$; все логарифмы далее берутся по основанию 2.

Сформулированный выше результат из [13] непосредственно переносится на прямоугольные укладки и клеточные схемы. Требование выпуклости существенно: $\boldsymbol{H}$-дерево [8] с $n$ листьями имеет сумму длин ребер и площадь укладки одновременно равные $\Theta(n)$. В [2] приведен пример такой последовательности булевых функий $g_{n}=g_{n}\left(x_{1}, \ldots x_{n}\right)$, что площадь реализации функции $g_{n}$ правильной клеточной схемой равна $\Theta\left(n^{3 / 2}\right)$, а неправильной (то есть такой, у которой входы не обязательно лежат на границе) - $\Theta(n)$. Эти результаты показывают, что правильная и неправильная клеточные модели существенно различны.

Результат из [13] уточнен в работе [5], где доказано, что наименьший линейный размер прямоугольной решетки, куда правильно 2-вложено полное двоичное дерево с $2^{n}$ листьями, равен $\lceil(n+1) / 2\rceil$. Там же построено вложение, у которого один из линейных размеров минимален, а площадь асимптотически равна $(1 / 4) n 2^{n}$ и доказана ее минимальность. Позднее С. А. Ложкин [6] установил аналогичный результат для площади 1 -вложений с асимптотическим значением площади, равным $(1 / 3) n 2^{n}$.

Н. А. Шкаликова [11] показала, что площадь каждой клеточной схемы, осуществляющей умножение двух $n$-разрядных чисел, заданных в двоичной системе счисления, не меньше $\Omega\left(n^{2}\right)$, и привела пример схемы площади $\Theta\left(n^{2}\right)$ и глубины $\Theta(n)$. В [3] приведен пример клеточной схемы для умножения с глубиной $\Theta(\log n)$ и площадью $\Theta\left(n^{2} \log n\right)$. Неизвестно, можно ли избавиться от множителя $\log n$ в оценке площади схемы для умножения, сохраняя глубину логарифмической. Однако для задачи сложения в более слабой модели клеточных $T$-схем можно доказать его необходимость (см. ниже).

Из результатов работ $[5,6,13]$ следует нижняя оценка $\Omega(n \log n)$ для площади клеточных схем, обладающих полными остовными деревьями с $n$ листьями по краям укладки. Для неполных деревьев общего вида нижние оценки площади укладки пока неизвестны. В данной статье предлагается модель клеточных $T$-схем. $T$-схема отличается тем, что расстояние между концами любого пути в ее укладке пропорционально числу изломов пути. В этой модели получена нижняя оценка площади правильной укладки произвольного, не обязательно полного, двоичного дерева (раздел 2). Приведены примеры задач (префиксные суммы и $\boldsymbol{n}$-разрядные сумматоры), схемы которых являются оптимальными в этой модели (раздел 3).

\section{2. Нижние оценки}

Теорема $1([5,13])$. Пусть $S$ - плоская прямоугольная укладка полного двоичного п-ярусного дерева с листьями по краям укладки. Тогда для площади и линейных размеров укладки $S$ справедливы оченки

$$
A(S)=\Omega\left(n 2^{n}\right), \quad l(S)=\Omega(n), \quad h(S)=\Omega(n) .
$$

Следствие 1. Для всякой клеточной схемы $S$ с $2^{n}$ входами, реализующей существенно зависячую от $2^{n}$ аргументов функчию и имеючей минимально возможную глубину $d(S)=n$, справедливы оченки плочади, длины и ширины

$$
A(S)=\Omega\left(n 2^{n}\right), \quad l(S)=\Omega(n), \quad h(S)=\Omega(n) .
$$


Рассмотрим теперь частный случай клеточных схем - клеточные $T$-схемы. Будем называть клеточный элемент $\boldsymbol{X}$ элементом верхнего (нижнего, левого, правого) направления, если на его верхней (соответственно нижней, левой или правой) границе расположен один из его входов. В противном случае будем говорить, что элемент $X$ не является элементом верхнего (нижнего, левого, правого) направления. Элементы без входов не являются элементами никакого из четырех направлений.

Определение 1. Пусть все входы правильной клеточной схемы $S$ расположены на ее нижней (верхней, левой, правой) границе, а выходы - на противоположной. Схему $S$ назовем схемой трех направлений или $T$-схемой, если все ее клеточные элементы не являются элементами верхнего направления (либо, соответственно, нижнего, правого или левого).

Например, в $T$-схеме, где все клеточные элементы не являются элементами верхнего направления, никакой ориентированный путь, ведущий снизу вверх от входа к выходу, не содержит отрезков с направлением сверху вниз (но может содержать горизонтальные отрезки). Иначе говоря, информация в такой схеме не может двигаться вниз.

Теорема 2. Всякая $T$-схема глубины $d>n$, которая реализует существенно зависящую от $2^{n}$ аргументов функцию, имеет площадь $\Omega\left(n 2^{n} / \log (d / n)\right)$.

Доказательство. Пусть $S-T$-схема с указанными в формулировке теоремы параметрами. Для определенности будем считать, что $S$ - схема не верхнего направления, то есть все входы расположены на ее нижней границе, выходы - на верхней, и среди составляющих ее клеточных элементов нет элементов со входами наверху. Площадь схемы есть произведение ее длины $l=l(S)$ и ширины $h=h(S)$, и $2^{n}$ входов расположены на горизонтальной части границы, поэтому $l=\Omega\left(2^{n}\right)$ и достаточно доказать, что

$$
h=\Omega\left(\frac{n}{\log (d / n)}\right) .
$$

Рассмотрим прямоугольную укладку $T$ схемы $S$. Без ограничения общности можно считать, что она является укладкой двоичного дерева. Если это не так, то возьмем какое-нибудь ориентированное к выходу остовное дерево схемы $S$, глубина которого не превышает глубины схемы, а корень является ее выходом. Ясно, что такое всегда найдется. Ему принадлежат все входы схемы, и ширина $h$ схемы $S$ не меньше чем ширина укладки такого дерева.

Пусть мы хотим попасть из корня дерева $S$ в какой-либо его лист, следуя в обратном направлении по ребрам прямоугольной решетки, принадлежащим укладке $T$. Пусть уже пройдена часть этого пути и мы находимся в некоторой вершине $v \in T$. Если $v-$ не функциональный элемент схемы $S$, то есть является промежуточной вершиной, то путь однозначно продолжается в единственную смежную вершину $v^{\prime} \in T$. Смежная вершина $v^{\prime}$ также определяется единственным образом, если $v$ - функциональный элемент, реализующий одноместную функцию. Если же $v$ - двуместный функциональный элемент, то в $v$ входят два ребра из некоторых вершин $v^{\prime}$ и $v^{\prime \prime}$ из $T$. Такую вершину $v$ назовем 2-вершиной. Чтобы определить, в какую из смежных ей вершин перейти, нужна дополнительная информация.

Каждому пути $\gamma$, ведущему от выхода-корня к какому-либо входу-листу укладки $T$, поставим в соответствие его код $c(\gamma)$ так, чтобы по $T$ (или $S$ ) и $c(\gamma)$ можно было однозначно найти $\gamma$. Так как глубина схемы равна $d$, на пути $\gamma$ лежит не более $d$ принадлежащих укладке 2-вершин. Для каждой такой вершины достаточно указать одно из двух 
направлений движения, по которому идет путь $\gamma$, чтобы задать его. Занумеруем подряд 2-вершины, через которые проходит $\gamma: v_{1}, v_{2}, \ldots, v_{N}$. Можно считать, что первым в этом списке стоит корень дерева - выход схемы $S$. Кодом $c(\gamma)$ будет набор $\left(c_{1}, c_{2}, \ldots, c_{N}\right)$, построенный по следующему принщипу. Пусть $c_{0}=0$. Добавим в укладку фиктивную вершину $v_{0}$ непосредственно над корнем $v_{1}$ и ведущее к ней из $v_{1}$ вертикальное ребро $\uparrow$. Предположим, что мы находимся в вершине $v_{i} \in T, i \in\{1, \ldots, N\}$, и нам уже известно значение $c_{i-1}$. В вершину $v_{i}$ входят два ориентированных ребра $e^{\prime}$ и $e^{\prime \prime}$, одно из которых принадлежит $\gamma$, другое нет. Пусть $e^{\prime} \in \gamma, e^{\prime \prime} \notin \gamma$ и пусть $e \in \gamma-$ ребро, входящее в вершину $v_{i-1}$. Раз дерево $S$ вложено в прямоугольную решетку и не является схемой верхнего направления, каждое из ребер $e, e^{\prime}, e^{\prime \prime}$ принадлежит одному из трех типов: $\uparrow$, $\rightarrow$, $\leftarrow$ и у ребер $e^{\prime}, e^{\prime \prime}-$ разный тип. Если тип ребра $e^{\prime}$ тот же, что и у ребра $e$, то положим $c_{i}=c_{i-1}$. Если это не так, то положим $c_{i}=c_{i-1}+1(\bmod 3)$ в случае когда измеренный по часовой стрелке угол между векторами $e$ и $e^{\prime}$ меньше, чем между $e$ и $e^{\prime \prime}$, и $c_{i}=c_{i-1}-1(\bmod 3)$, когда угол между $e$ и $e^{\prime \prime}$ меньше угла между $e$ и $e^{\prime}$ :

$$
c_{i}=\left\{\begin{array}{lll}
c_{i-1}, & \text { если }\left(e, e^{\prime}\right) \in\{(\uparrow, \uparrow),(\rightarrow, \rightarrow),(\leftarrow, \leftarrow)\}, \\
c_{i-1}+1 & (\bmod 3), & \text { если }\left(e, e^{\prime}, e^{\prime \prime}\right) \in\{(\uparrow, \rightarrow, \leftarrow),(\rightarrow, \leftarrow, \uparrow),(\leftarrow, \uparrow, \rightarrow)\}, \\
c_{i-1}-1 & (\bmod 3) & \text { в противном случае }
\end{array}\right.
$$

Таким образом, каждому пути $\gamma$ с $N$ 2-вершинами соответствует троичный код $c(\gamma)$ длины $N$, причем по укладке на плоскости и коду путь в укладке и вход схемы, которым он оканчивается, находятся однозначно. Более того, если $c_{i} \neq c_{i+1}$, то заключенный между вершинами $v_{i}$ и $v_{i+1}$ отрезок пути $\gamma$ имеет хотя бы один излом, то есть хотя бы единожды на этом участке $\gamma$ меняет направление. Центральным в доказательстве является следующее наблюдение: из того, что число изломов ориентированного пути равно $k$ и путь состоит только из отрезков вида $\uparrow, \rightarrow$ и $\leftarrow$, следует, что расстояние по вертикали между его концами, то есть ширина пути, не меньше чем $\lfloor k / 2\rfloor$.

Пусть $k=k(\gamma)$ - число пар различных соседних чисел $c_{i}$ в наборе $c(\gamma)$. Тогда путь $\gamma$ содержит не менее $k$ изломов и его ширина не меньше чем $\{k / 2\rfloor$. Легко видеть, что число кодовых наборов $c(\gamma)$ длины $N$, у которых ровно $k$ пар соседних разрядов $\left(c_{i}, c_{i+1}\right)$ различаются на единицу, не превышает $\left(\begin{array}{c}N \\ k\end{array}\right) 2^{k}$. Действительно, в наборе $\left(c_{0}, c_{1}, \ldots c_{N}\right)\left(\begin{array}{l}N \\ k\end{array}\right)$ способами можно выбрать $k$ разрядов $i_{1}, \ldots i_{k}$ так, чтобы $c_{i_{j}} \neq c_{i_{j}+1}$. Зная $c_{i_{j}}$, значение следующего числа $c_{i_{j}+1}$ можно выбрать двумя способами: оно отличается от $c_{i j}$ ровно на единицу. Чтобы найти количество наборов $\left(c_{1}, \ldots c_{N}\right)$, равное количеству наборов $\left(0, c_{1}, \ldots c_{N}\right)$, надо числа всех этих способов перемножить.

Общее количество путей $\gamma$ от корня к листьям в укладке дерева $S$ глубины $d$ и ширины $\lfloor k / 2\rfloor$ не превышает количества кодовых наборов $c(\gamma)$ длины, не большей $d, \mathrm{c}$ не более чем $k$ парами различных соседних разрядов. Это количество в свою очередь не превышает $\sum_{l \leqslant k} \sum_{N \leqslant d}\left(\begin{array}{l}N \\ l\end{array}\right) 2^{l}$.

Различных путей от корня к листьям в дереве столько же, сколько и листьев, то есть $2^{n}$. Поэтому если у дерева глубины $d$ с $2^{n}$ листьями ширина $h$ его укладки в прямоугольную решетку не превосходит $\lfloor k / 2\rfloor$, то должно выполняться неравенство

$$
\sum_{l \leqslant k} \sum_{N \leqslant d}\left(\begin{array}{l}
N \\
l
\end{array}\right) 2^{l} \geqslant 2^{n} .
$$

Нас интересует наибольшее возможное $k$, при котором неравенство (2) будет оставаться неверным. Вначале рассмотрим крайний случай: при каких $d$ выбор $k=O(1)$ при 
$n \rightarrow \infty$ не будет противоречить неравенству (2)? При $k$ фиксированном очевидно, что $k \ll d$; при $l \leqslant k, N \leqslant d$ для биномиальных коэффициентов справедливы неравенства

$$
\left(\begin{array}{l}
N \\
l
\end{array}\right) \leqslant\left(\begin{array}{l}
d \\
l
\end{array}\right) \leqslant\left(\begin{array}{l}
d \\
k
\end{array}\right)
$$

Следовательно,

$$
\sum_{l \leqslant k} \sum_{N \leqslant d}\left(\begin{array}{l}
N \\
l
\end{array}\right) 2^{l} \leqslant \sum_{l \leqslant k} d\left(\begin{array}{l}
d \\
l
\end{array}\right) 2^{l} \leqslant d\left(\begin{array}{l}
d \\
k
\end{array}\right) 2^{k+1} .
$$

Воспользовавшись (3) и учитывая, что

$$
\left(\begin{array}{l}
d \\
k
\end{array}\right) \leqslant d^{k}
$$

из неравенства (2) получим, что

$$
d^{k+1} \geqslant 2^{n-k-1}
$$

откуда следует, что

$$
d \geqslant 2^{(n-k-1) /(k+1)}
$$

Мы получили, что $h=\Omega(1)$ тогда и только тогда, когда $d=2^{\Omega(n)}$. Это частный случай утверждения (1). Поэтому в дальнейшем мы вправе считать $k$ неограниченным с ростом $n$ и одновременно $d=2^{o(n)}$.

Предположим, что

$$
h=o(n / \log (d / n))
$$

при $n \rightarrow \infty$. Это равносильно тому, что

$$
k=o(n / \log (d / n)) .
$$

Так как глубина схемы, реализующей существенно зависящую от всех своих $2^{n}$ аргументов функцию не может быть меньше $n$, получаем, что

$$
d=d(n)=\Omega(n)
$$

и из нашего предположения следует, что

$$
k=o(n)=o(d)
$$

Оценка (3) остается в силе, и из неравенства (2) вытекает, что

$$
d\left(\begin{array}{l}
d \\
k
\end{array}\right) 2^{k+1} \geqslant 2^{n}
$$


Так как $k$ и $d$ вместе растут с ростом $n$, можно воспользоваться формулой Стирлинга и вытекающим из нее асимптотическим приближением биномиального коэффищиента

$$
\left(\begin{array}{l}
d \\
k
\end{array}\right) \sim \sqrt{\frac{d}{2 \pi k(d-k)}} \frac{d^{d}}{k^{k}(d-k)^{d-k}} .
$$

Прологарифмировав обе части неравенства (4), получим, что

$\log d+\log \sqrt{\frac{d}{2 \pi k(d-k)}}+d \log d-k \log k-(d-k) \log (d-k)+k+1-n+o(1) \geqslant 0$, откуда, учитывая, что

$$
d \log d-k \log k-(d-k) \log (d-k)=d \log \frac{d}{d-k}+k \log \frac{d-k}{k},
$$

приходим к соотношению

$$
d \log \frac{d}{d-k}+k \log \frac{d-k}{k}+O(\log d)+k-n \geqslant 0 .
$$

Разделим обе части на $n$ и получим, что для существования укладки с шириной $\lfloor k / 2\rfloor$ необходимо выполнение неравенства

$$
\frac{d}{n} \log \frac{d}{d-k}+\frac{k}{n} \log \frac{d-k}{k}+O\left(\frac{\log d}{n}\right)+\frac{k}{n}-1 \geqslant 0 .
$$

Рассмотрим его слагаемые по отдельности. Во-первых,

$$
\frac{d}{n} \log \frac{d}{d-k}=\frac{d}{n} \log \left(1+O\left(\frac{k}{d}\right)\right)=\frac{d}{n}\left(O\left(\frac{k}{d}\right)+o\left(\frac{k}{d}\right)\right)=O\left(\frac{k}{n}\right),
$$

так как $k=o(d)$. Далее, раз $k=o(r / \log (d / n)$ и $k$ не равно постоянной, его можно представить в виде $k=n / m$, где $m \rightarrow \infty$ и $m / \log (d / n) \rightarrow \infty$, и второе слагаемое из (5) можно оценить так:

$$
\frac{k}{n} \log \frac{d-k}{k} \leqslant \frac{k}{n} \log \frac{d}{k}=\frac{1}{m} \log \frac{d m}{n}=\frac{\log \frac{d}{n}}{m}+\frac{\log m}{m}=o(1) .
$$

Наконец, в силу очевидных оценок

$$
O(k / n)=O(o(n / \log (d / n)) / n)=o(1 / \log (d / n))=o(1)
$$

И

$$
O\left(\frac{\log d}{n}\right)=O\left(\frac{\log 2^{o(n)}}{n}\right)=\frac{o(n)}{n}=o(1),
$$

из неравенств (5), (6) и (7), следует, что $o(1)-1 \geqslant 0$ при $n \rightarrow \infty$. Мы получили противоречие, предположив, что $h=o(n / \log (d / n))$ и взяв $k$ наименьшим таким, что $h=\lfloor k / 2\rfloor$. Значит,

$$
h=\Omega(n / \log (d / n))
$$

для достаточно больших $n$.

Следствие 2. Каждая T-схема, которая реализует существенно зависящую от $n$ аргументов функцию с глубиной $O(\log n)$, имеет площадь по порядку не меньшую, чем $n \log n$. 


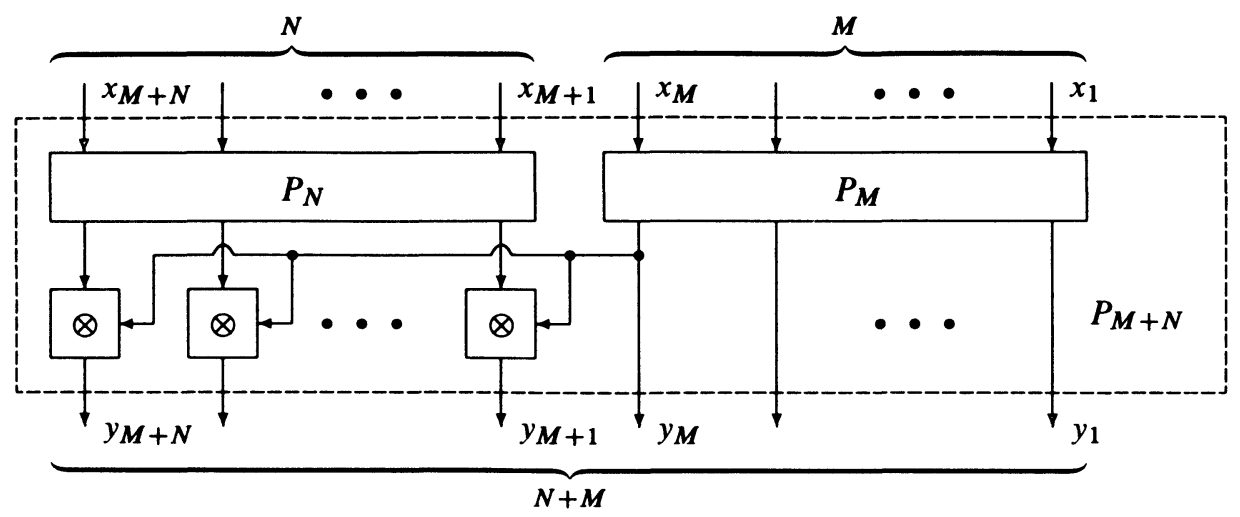

Рис. 2. Индуктивное построение префиксной схемы

\section{3. Примеры оптимальных $T$-схем}

\section{1. Префиксные клеточные схемы}

Задача вычисления префиксной суммы $[12,15]$ заключается в следующем. Пусть имеется ассоциативная операция $\otimes$ на некотором множестве $M$ и набор элементов $x_{1}, x_{2}, \ldots, x_{n}$ из этого множества. Требуется найти набор величин $y_{1}, y_{2}, \ldots, y_{n}$, где

$$
y_{k}=\bigotimes_{i=1}^{k} x_{i}, \quad 1 \leqslant k \leqslant n .
$$

Величина $y_{k}$ называется $k$-й префиксной суммой последовательности $\left\{x_{i}\right\}$.

Определение 2. Назовем префиксной клеточную схему с $n$ входами $x_{1}, x_{2}, \ldots, x_{n}$ и $n$ выходами $y_{1}, y_{2}, \ldots, y_{n}$, если каждый функциональный элемент схемы реализует операцию $\otimes$ и схема вычисляет префиксные суммы входных значений $y_{k}=\bigotimes_{i=1}^{k} x_{i}$ при всех $k, 1 \leqslant k \leqslant n$.

Площадь $A(S)$, длина $l(S)$, ширина $h(S)$ и глубина $d(S)$ клеточной схемы $S$ с функциональными элементами $\otimes$ определяются аналогично соответствующим характеристикам схемы из $k$-ичных функщинальных элементов. С помощью стандартной техники [12] нетрудно доказать следующее утверждение.

Теорема 3. Для всякого натурального числа п существует префиксная схема $P_{n}$ со входами $x_{1}, x_{2}, \ldots x_{n}$ и выходами $y_{1}, y_{2}, \ldots y_{n}$, для глубины и площади которой справедливы оченки

$$
\begin{aligned}
& d\left(P_{n}\right)=\lceil\log n\rceil, \\
& A\left(P_{n}\right) \leqslant 4 n\lceil\log n\rceil .
\end{aligned}
$$

Доказательство. Пусть известны схемы $P_{M}$ и $P_{N}$, вычисляющие префиксные суммы наборов переменных $x_{1}, \ldots, x_{M}$ и $x_{M+1}, \ldots, x_{M+N}$ соответственно. Тогда из них можно построить схему $P_{M+N}$, находящую префиксные суммы объединения этих наборов, что иллюстрируется на рис. 2 . 
Нетрудно видеть, что ее глубина удовлетворяет неравенству

$$
d\left(P_{M+N}\right) \leqslant \max \left(d\left(P_{M}\right), d\left(P_{N}\right)\right)+1,
$$

а длина и ширина - неравенствам

$$
\begin{aligned}
& l\left(P_{M+N}\right) \leqslant l\left(P_{M}\right)+l\left(P_{N}\right), \\
& h\left(P_{M+N}\right) \leqslant \max \left(h\left(P_{M}\right), h\left(P_{N}\right)\right)+2 .
\end{aligned}
$$

Легко также убедиться, что существует схема $P_{2}$ с параметрами

$$
d\left(P_{2}\right)=1, \quad l\left(P_{2}\right)=4, \quad h\left(P_{2}\right)=2 .
$$

Пусть $n=2^{m}$. Тогда, положив

$$
M=N=\frac{n}{2},
$$

из неравенств (8), (9) и (10) получим, что

$$
d\left(P_{2^{m}}\right) \leqslant m, \quad l\left(P_{2^{m}}\right) \leqslant 2 \cdot 2^{m}, \quad h\left(P_{2^{m}}\right) \leqslant 2 m .
$$

Если же $n \neq 2^{m}$, то возьмем $M=2^{m}$, так, чтобы $2^{m}<n<2^{m+1}$. Положим $N=n-M$. Заметим, что $d\left(P_{i}\right) \leqslant d\left(P_{j}\right), h\left(P_{i}\right) \leqslant h\left(P_{j}\right)$ при $i \leqslant j$. Тогда

$$
d\left(P_{n}\right) \leqslant d\left(P_{M}\right)+1=m+1=\lceil\log n\rceil .
$$

Неравенства (9) и (10) в свою очередь преобразуются к виду

$$
\begin{aligned}
& l\left(P_{n}\right) \leqslant l\left(P_{N}\right)+2 \cdot 2^{m}, \\
& h\left(P_{n}\right) \leqslant h\left(P_{M}\right)+2=2 m+2=2\lceil\log n\rceil .
\end{aligned}
$$

Представив $n$ в двоичной системе счисления, получим, что

$$
n=\sum_{i=1}^{l} 2^{m_{i}}
$$

где

$$
m_{1}=m>m_{2}>\ldots>m_{l}, \quad l \leqslant\lceil\log n\rceil .
$$

Из (11) по индукщии находим, что

$$
l\left(P_{n}\right) \leqslant 2 \sum_{i=1}^{l} 2^{m_{i}}=2 n .
$$

Теорема доказана.

Замечание 1. Легко убедиться, что построенная схема $P_{n}$ имеет минимально возможную глубину и в ней нет элементов нижнего направления. Из теорем 1 и 2 следует, что у схемы $P_{n}$ минимальна не только глубина, но и одновременно минимальна по порядку площадь. Точнее, всякая префиксная схема с $n$ входами и минимальной глубиной $\lceil\log n\rceil$ имеет площадь $\Omega(n \log n)$. Про $T$-схемы можно сказать больше: всякая префиксная $T$-схема с $n$ входами и глубиной $O(\log n)$ имеет площадь $\Omega(n \log n)$. 


\section{2. Клеточные $k$-ичные сумматоры}

Пусть заданы два $n$-разрядных числа $X$ и $Y$, записанные в позиционной системе счисления с основанием $k \geqslant 2$

$$
X=\sum_{i=1}^{n} x_{i} k^{i-1}, \quad Y=\sum_{i=1}^{n} y_{i} k^{i-1}, \quad x_{i}, y_{i} \in\{0,1, \ldots, k-1\}
$$

В этом разделе мы построим клеточную схему, определяющую разряды суммы

$$
Z=X+Y=\sum_{i=1}^{n+1} z_{i} k^{i-1}
$$

В дальнейшем такую схему будем называть (клеточным) $k$-ичным $n$-разрядным сумматором. Очередной разряд $z_{i}$ суммы $Z$ можно получить из соответствующих разрядов $x_{i}$ и $y_{i}$ слагаемых $X$ и $Y$, учитывая перенос $w_{i}$ из предыдущего $(i-1)$-го разряда в следующий $i$-й разряд, по формулам

$$
\begin{aligned}
z_{i} & =x_{i} \oplus y_{i} \oplus w_{i}, \\
w_{i} & =\left(x_{i-1} \&_{k} y_{i-1}\right) \vee w_{i-1} \&\left(x_{i-1} \vee_{k} y_{i-1}\right)
\end{aligned}
$$

Здесь $\bigoplus$ обозначает сложение по модулю $k, \&-$ пороговую функцию, равную 1 , если сумма аргументов превышает $k-1$ и 0 в противном случае, $V$ - пороговую функцию, равную 1 , если сумма аргументов превышает $k-2$, то есть

$$
\begin{aligned}
& x \& y= \begin{cases}1, & \text { если } x+y \geqslant k, \\
0 & \text { в противном случае, }\end{cases} \\
& x \vee y=\left\{\begin{array}{l}
1, \text { если } x+y \geqslant k-1, \\
\text { 0в противном случае. }
\end{array}\right.
\end{aligned}
$$

Знаки \& и $\vee$ обозначают традиционные конъюнкцию и дизъюнкцию (отметим, что при всяком $k$ перенос из разряда в разряд не может превышать единицу, то есть он - булева величина). Будем считать, что $w_{1}=0$.

Н. А. Шкаликова в [11] привела пример клеточного двоичного $n$-разрядного сумматора площади $\Theta(n)$ и глубины $\Theta(n)$. Аналогично можно построить и $k$-ичный сумматор с площадью и глубиной $\Theta(n)$ для $k \geqslant 3$. Очевидно, что площадь таких сумматоров оптимальна по порядку. Глубина, однако, далека от минимально возможной.

Известно $[9,16]$, что в модели схем из функциональных элементов существуют $n$-разрядные сумматоры, у которых глубина и сложность оптимальны по порядку одновременно, глубина равна $\Theta(\log n)$, а сложность $-\Theta(n)$. Из нижних оценок теорем 1 и 2 вытекает, что в модели клеточных $T$-схем это невозможно: всякая схема глубины $O(\log n)$ имеет площадь $\Omega(n \log n)$. Иначе говоря, теорему 2 можно рассматривать как утверждение о невозможности одновременно оптимизировать и площадь, и глубину в классе клеточных $T$-схем. 
Теорема 4. При $k \geqslant 3$ существует $k$-ичный n-разрядный клеточный сумматор $\Sigma_{n}$ глубины

$$
d\left(\Sigma_{n}\right) \leqslant\left\lceil\log _{2}(n+1)\right\rceil+2 .
$$

Его площадь удовлетворяет неравенству

$$
A\left(\Sigma_{n}\right) \leqslant 6 n\left(\left\lceil\log _{2}(n+1)\right\rceil+2\right)
$$

и минимальна по порядку в классе Т-схем логарифмической глубины.

Доказательство. Пусть для простоты $k=3$ (при бо́льших $k$ все рассуждения аналогичны). Сумматор логарифмической глубины основан на хорошо известной конструкщии под названием “предвычисление переноса" (см. [12, 16]). Если в формуле (14) известны перенос $w_{i}$ и величина $x_{i} \oplus y_{i}$, то разряды суммы $z_{i}$ для всех $i$ можно вычислить подсхемой глубины 1 , и задача сводится к нахождению быстрой схемы для $w_{i}$.

Каждая пара разрядов слагаемых $x_{i}$ и $y_{i}$ характеризуется числом $\chi_{i}$, равным 0,1 или 2. Паре $\left(x_{i}, y_{i}\right)$ сопоставим $\chi_{i}=0$, если $x_{i}+y_{i} \leqslant 1$. Такая пара разрядов не создает перенос в следующий разряд, даже если предыдущая пара дала перенос в $i$-й разряд (то есть $w_{i+1}=0$ при любом $w_{i}$ ). Двойка сопоставляется паре, если $x_{i}+y_{i}=2$. Такая пара при сложении передаст перенос из предыдущего в следующий разряд (то есть $w_{i+1}=1$ тогда и только тогда, когда $\left.w_{i}=1\right)$. Наконец, в случае $x_{i}+y_{i} \geqslant 3$ сопоставим паре $\left(x_{i}, y_{i}\right)$ число $\chi_{i}=1$, в этом случае перенос $w_{i+1}$ равен единице вне зависимости от $w_{i}$. Величину $\chi_{i}$ назовем характеристикой $i$-го разряда.

Определить для разряда с номером $i$, чему равен перенос $w_{i}$, можно с помощью характеристик предыдущих разрядов. Предыдушие разряды создадут перенос $w_{i}=1$, если первая характеристика (начиная с $\chi_{i-1}$ ) среди характеристик младших разрядов $\chi_{i-1}, \ldots, \chi_{2}, \chi_{1}$, отличная от 2 , равна 1 . Аналогично, переноса не будет $\left(w_{i}=0\right)$, если первая отличная от 2 характеристика младшего разряда равна 0.

Рассмотрим две функщии трехзначной логики $x \odot y$ и $x \otimes y$ :

$$
\begin{aligned}
& x \odot y=\left\{\begin{array}{l}
0, \text { если } x+y \leqslant 1, \\
1, \text { если } x+y \geqslant 3, \\
2, \text { если } x+y=2,
\end{array}\right. \\
& x \otimes y=\left\{\begin{array}{l}
x, \text { если } x=0 \text { или } x=1, \\
y, \text { если } x=2 .
\end{array}\right.
\end{aligned}
$$

Легко видеть, что функция $\otimes$, понимаемая как операция на множестве $\{0,1,2\}$, ассоциативна, то есть

$$
x \otimes(y \otimes z)=(x \otimes y) \otimes z
$$

для произвольных $x, y, z$. В силу сказанного выше, $\chi_{i}=x_{i} \odot y_{i}$, а перенос $w_{i}$ можно вычислить по формуле

$$
w_{i+1}=\chi_{i} \otimes \chi_{i-1} \otimes \ldots \otimes \chi_{1} \otimes 0, \quad i=1,2, \ldots, n
$$

(здесь мы учли, что $w_{1}=0$ ). Из равенства (15) заключаем, что вычисление совокупности переносов $\left\{w_{i}\right\}_{i=2}^{n+1}$ сводится к нахождению префиксных сумм характеристик $\chi_{n}, \ldots, \chi_{1}$ по отношению к операции $\otimes$. Для этого можно применить какую-нибудь префиксную схему. Полученные на ее выходах переносы сложим с разрядами слагаемых одновременно и независимо, и найдем $z_{i}$. 
Таким образом, клеточный сумматор $\Sigma_{n}$ состоит из трех подсхем $A, B, C$. Подсхема $A$ находит характеристики $\chi_{i}=x_{i} \odot y_{i}$ и суммы $x_{i} \oplus y_{i}$ всех пар разрядов, $1 \leqslant i \leqslant n$. Подсхема $B$ является экземпляром схемы $P_{n+1}$ из теоремы 3 , на ее входы подаются выходы $\chi_{i}$ схемы $A$ и константа 0 . У схемы $B$ есть также $n$ вспомогательных входов, с которых величины $x_{i} \oplus y_{i}$ поступают к вспомогательным выходам, соединенным с подсхемой $C$. Наконец, подсхема $C$ независимо вычисляет разряды суммы $Z=X+Y$ по формуле (14). Пример 7-разрядного сумматора $\Sigma_{7}$ приведен на рис. 3.

Оценим параметры схемы $\Sigma_{n}$. Ясно, что

$$
d(A)=d(C)=1, \quad d(B)=\left\lceil\log _{2}(n+1)\right\rceil .
$$

Как нетрудно убедиться, схемы $A, B, C$ можно соединить так, что

$$
\begin{gathered}
l\left(\Sigma_{n}\right)=l(A)=l(B)=l(C)=3(n+1), \\
h(A)=3, \quad h(B)=h\left(P_{n+1}\right) \leqslant 2\left\lceil\log _{2}(n+1)\right\rceil, \quad h(C)=1 .
\end{gathered}
$$

Отсюда получаем оценки глубины

$$
\begin{aligned}
d\left(\Sigma_{n}\right) & =d(A)+d(B)+d(C) \\
& \leqslant\left\lceil\log _{2}(n+1)\right\rceil+2,
\end{aligned}
$$

и площади

$$
\begin{aligned}
A\left(\Sigma_{n}\right) & =l\left(\Sigma_{n}\right) h\left(\Sigma_{n}\right)=3(n+1)(h(A)+h(B)+h(C)) \\
& \leqslant 3(n+1)\left(2\left\lceil\log _{2}(n+1)\right\rceil+4\right) \\
& =O(n \log n) .
\end{aligned}
$$

Очевидно, что в полученной схеме $\Sigma_{n}$ нет элементов нижнего направления. В классе $T$-схем оптимальность ее площади с точностью до постоянного множителя вытекает из следствия теоремы 2.

В случае $k=2$ размер разряда слишком мал, чтобы учесть все три альтернативы для характеристики пары разрядов слагаемых - создавать перенос, обнулять и передавать из предыдущего разряда. Каждое из ее возможных значений $0,1,2$ можно закодировать парой битов. Нетрудно построить двоичную клеточную $T$-схему размера $4 \times 4$ и глубины 2, моделирующую троичный клеточный элемент $\otimes$. Заменяя все клеточные элементы троичного сумматора $\Sigma_{n}$ на соответствующие двоичные схемы (с очевидным изменением подсхемы $A$ ), получим двоичный сумматор $\Sigma_{n}^{2}$. По построению

$$
\begin{aligned}
& d\left(\Sigma_{n}^{2}\right)=2 d\left(\Sigma_{n}\right), \\
& A\left(\Sigma_{n}^{2}\right)=16 A\left(\Sigma_{n}\right) .
\end{aligned}
$$

Следствие 3. Существует двоччный п-разрядный клеточный сумматор алубины $2 \log _{2} n+O(1)$ и плочади $\Theta(n \log n)$, минимальной по порядку в классе $T$-схем логарифмической глубины. 


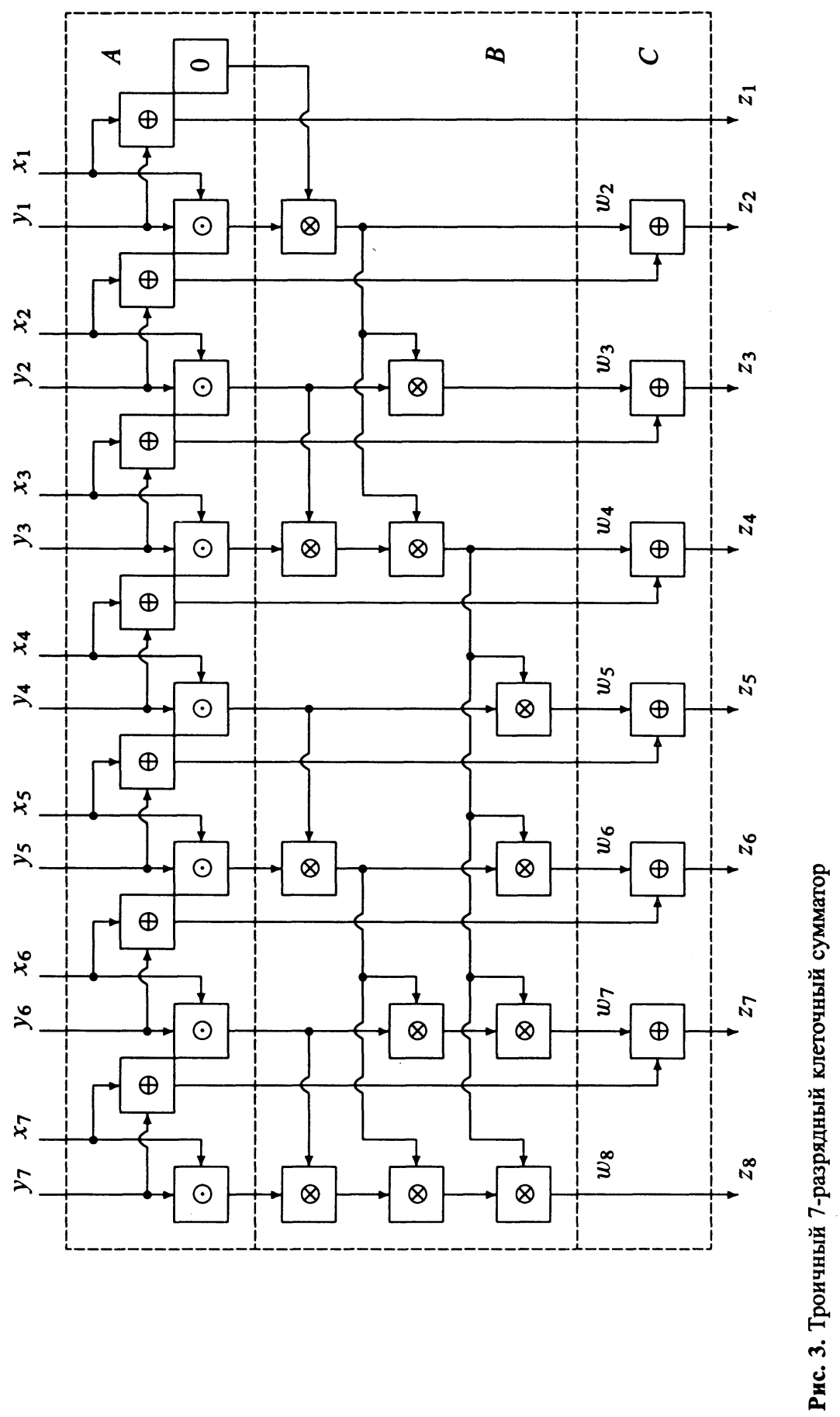


В работе А. В. Чашкина [10] описан простой способ определения разности двоичных $n$-разрядных чисел с помощью сумматора. Его можно обобщить на $k$-ичный случай, когда $k \geqslant 3$. Пусть числа $X$ и $Y$ записаны в системе счисления с основанием $k$ (см. (13)), и пусть $X \geqslant Y$. Обозначим через $\bar{X}$ такое число, что $X+\bar{X}=k^{n}-1$. Иначе говоря,

$$
\bar{X}=\sum_{i=1}^{n} \bar{x}_{i} k^{i-1},
$$

где $\bar{x}_{i}$ обозначает отрицание $i$-го разряда числа $X$ по Лукашевичу: $\bar{x}=k-1-x$.

Очевидно, что

$$
X-Y=\bar{X}+Y \text {. }
$$

Следовательно, если заранее известно, что разность неотрицательна, то для ее вычисления можно использовать любой сумматор. Достаточно обратить разряды уменьшаемого числа $X$ и разряды результата $\bar{X}+Y$.

Следствие 4. Для $k \geqslant 3$ существует клеточная $k$-ичная $T$-схема $\bar{\Sigma}_{n}$, находящая разность п-разрядных чисел $X$ и $Y$ при условии, что $X \geqslant Y$, для глубины которой справедливо равенство

$$
d\left(\bar{\Sigma}_{n}\right)=\log _{2} n+O(1)
$$

В базисе из всех двоичных клеточных элементов существует схема вычитания глубины $2 \log _{2} n+O(1)$. Плочади этих схем равны $\Theta(n \log n)$ и минимальны по порядку в классе Т-схем логарифмической глубины.

В заключение автор благодарит А. В. Чашкина за постановку задач и постоянное внимание к работе.

\section{Список литературы}

1. Альбрехт А., О схемах из клеточных элементов. Проблемы кибериетики (1977) 33, 209-214.

2. Громкович Ю., Шустер Б., О соотношении сложностей двух видов плоских схем из функциональных элементов. Дискретиая математика (1990) 2, №2, 121-126.

3. Жуков Д. А., Быстрые клеточные схемы для умножения. Дискретный анализ и исследование операчий (2002) 9, №3, 40-47.

4. Кравцов С. С., О реализации функций алгебры логики в одном классе схем из функциональных и коммутационных элементов. Проблемы кибериетики (1967) 19, 285-293.

5. Ложкин С. А., Ли Да Мин, О некоторых оптимальных вложениях двоичных и троичных деревьев в плоские прямоугольные решетки. Вестиик Московского университета, сер. 15 (1995) №4, 49-55.

6. Ложкин С. А., Асимптотически оптимальные гомеоморфные вложения полных двоичных и троичных деревьев в плоские прямоугольные решетки. В сб.: Проблемы теоретической киберпетики. Тез. докл. 13 Междупар. коиф. в Казапи, часть ІІ. Изд-во Центра прикладных исследований при мех.-мат. ф-те МГУ, Москва, 2002, с. 113.

7. Лупанов О. Б., Асимптотические очепки сложлости управляючих систем. Изд-во МГУ, Москва, 1984.

4 Дискретная математика, т.18 №4 
8. У'льман Дж. Д., Вычислительные аспекты СБИС. Радио и связь, Москва, 1990.

9. Храпченко В. М., Об асимптотической оценке времени сложения параллельного сумматора. Проблемы кибернетики (1967) 19, 107-122.

10. Чашкин А. В., Быстрое сложение и умножение целых чисел. В кн: Дискретиая математика и ее приложения: сборник лекций. II. Изд-во Центра прикладных исследований при мех.-мат. ф-те МГУ, Москва, 2001, с. 91-110.

11. Шкаликова Н. А., О реализации булевых функций схемами из клеточных элементов. Матем. вопросы кибернетики (1989) 2, 177-197.

12. Akl S. G., Parallel computation: models and methods. Prentice-Hall, New Jersey, 1997.

13. Brent R. P., Kung H. T., On the area of binary tree layouts. Inform. Process. Lett. (1980) 11, №1, 46-48.

14. Hromkovič J., Ložkin S. A., Rybko A. I., Sapoženko A. A., Škalikova N. A., Lower bounds on the area complexity of Boolean circuits. Theoret. Computer Sci. (1992) 97, №2, 285-300.

15. Ladner R. E., Fischer M. J., Parallel prefix computation. J. ACM (1980) 27, 831-838.

16. Wegener I., The complexity of Boolean functions. Teubner, Stuttgart, 1987.

Статья поступила 22.07.2003. 